\title{
A Recursive TF-ISF Based Sentence Retrieval Method with Local Context
}

\author{
Alen Doko, Maja Štula, and Darko Stipaničev
}

\begin{abstract}
Sentence retrieval consists of retrieving relevant sentences from a document base in response to a query. Question answering, novelty detection, summarization, opinion mining and information provenance make use of sentence retrieval. Most of the sentence retrieval methods are trivial adaptations of document retrieval methods. However some newer sentence retrieval methods based on the language modeling framework successfully use some kind of context of sentences. Unlike that there is no successful improvement of the TF-ISF method that takes into account the context of sentences. In this paper we propose a recursive TF-ISF based method that takes into account the local context of a sentence. The context is considered the previous and next sentence of current sentence. We compared the new method to the TF-ISF baseline and to an earlier unsuccessful method that also incorporates a similar context into TF-ISF. We got statistically significant improvements of the results in comparison to both of the methods. Additional benefit of our method is the clear explicit model of the context that will allow us to automatically generate a document representation with context suitable for sentence retrieval which is important for our future work.
\end{abstract}

Index Terms - Context, document representation, TF-ISF, sentence retrieval, recursion.

\section{INTRODUCTION}

Sentence retrieval is the task of finding relevant sentences from a document base in response to a query. Tasks like novelty detection, question answering, summarization, opinion mining and information provenance make use of sentence retrieval [1], [2]. Sentence retrieval methods are usually simple adaptations of document retrieval methods where sentences are treated as documents [3]-[5]. The state of the art and most successful models for sentence retrieval are the vector space model [1], [6], [7] and the language model approach with local context [1], [2]. The vector space model for sentence retrieval uses the term frequency - inverse sentence frequency method (TF-ISF) which is analogous to the traditional term frequency - inverse document frequency method (TF-IDF) used for document retrieval. When it comes to the language model approach for sentence retrieval at first it was implemented as a trivial adaptation from document retrieval to sentence retrieval, with sentences treated as documents [7]. In the recent works language model approach was successfully modified to take into account the context of sentence, like the document which contains it or some number of previous or next sentences [1], [2], [8]. The

Manuscript received January 5, 2013; revised April 19, 2013.

A. Doko is with the JP Croatian Telecommunications d.o.o. Mostar, Mostar, Bosnia and Herzegovina (e-mail: alen.doko@hteronet.ba)

M. Štula and D. Stipaničev are with the Faculty of Electrical Engineering, Mechanical Engineering and Naval Architecture, University of Split, Split, Croatia (e-mail: maja.stula@fesb.hr; darko.stipanicev@fesb.hr).
TF-ISF method was also modified to take into account context, but unsuccessfully without statistically significant improvements [1]. Fernandez sees the reason for using context of sentences in the vocabulary mismatch problem rooted in the sparsity of sentences [1]. He also thinks that the context of sentences contains a content that is not encoded in the sentence, but that is related to the sentence and it is important not just because of vocabulary mismatch. In other words the topic of the sentence is spread across the sentence and the context of the sentence. We agree that it is important to take into account local context of sentences if we want to develop good sentence retrieval methods. We especially think that it is valuable to improve the TF-ISF baseline which showed good results in the past [6], [8], [10] with the sentence's context. For that reason we make a new attempt to improve the TF-ISF method, explained in Section III to take into account the context of sentences.

\section{RELATED WORK}

Sentence retrieval methods are usually simple adaptations of document retrieval methods where sentences are treated as documents [3]-[5]. One of the first and most successful methods for sentence retrieval was the TF-ISF method [6] which is a trivial adaptation of the TF-IDF method for document retrieval. This method was shown to outperform other methods like BM25 based methods or language modeling based methods [6], [8], [10]. Another typically used method for sentence retrieval is the query likelihood method which is a language modeling approach to document retrieval. That method, invented by Ponte \& Croft for document retrieval [11] was in a trivial manner adapted and often used for sentence retrieval [2]. The TF-ISF and the query likelihood method can be considered baseline methods for sentence retrieval. The TF-ISF method and the query likelihood method were used as the baseline methods for sentence retrieval in [1] and the query likelihood method was used as the baseline method for sentence retrieval in [2].

In the recent works [1], [2] query likelihood was modified to take into account the local context of the sentence. Due to the sparsity of the sentences there is little overlap between the query and the sentence which negatively affects the performance of sentence retrieval [1]. The assumption is that this problem can be partially solved by using the local context of sentences.

The idea that "good" sentences come from "good" documents was proposed by Murdock [2]. So the query likelihood method was improved using local context in form of the document the sentence came from. A mixture model was proposed which combines a sentence language model, document language model and collection language model. 
The method showed better results when compared to the query likelihood baseline [2].

A sentence local context was also incorporated into the language modeling framework to better estimate the relevance of a sentence by Fernandez et. al. [1]. The document that contains the sentence and surrounding sentences (previous, current and next sentence) were used as the local context. Additionally, the importance of a sentence within a document was used. Tests showed significant improvements of language modeling methods when using local context in comparison to baselines like TF-ISF, and BM25 and to language modeling methods that ignore local context. Including sentence importance additionally improved the performance of tested language modeling approaches.

Fernandez et. al. also tried to improve the TF-ISF method by modifying it to take into account the local context [1]. Two types of local context, document that contains the sentence and surrounding sentences (previous, current and next sentence), were tested again. They tried to modify the TF part to take into account the number of occurrences of term in the context and also tried to compute the ISF part at document level rather than at sentence level. The tests did not show consistent and significant improvements.

A local context, implemented as a sliding window, was also used in [12] for the task of information retrieval. A sliding window consisted of multiple sentences. The whole sliding window was compared to the topic and if the sliding window is relevant all sentences inside it are considered relevant. The retrieval method was based on comparing nouns and verbs in the sliding window and the topic. Some tests showed best result when the sliding window is of size 4 but no comparisons were made to the state of the art methods.

\section{TF-ISF WITH LOCAL CONTEXT}

From the previous examples we saw that several sentence retrieval methods were improved by using of local context of sentences. One exception is the TF-ISF method. In this paper we show that it is possible to improve the TF-ISF method by using local context that consists of the neighboring sentences (previous and next sentence of the current sentence) and using a recursive ranking function. While in [1] it was tried to include the local context by modifying parts of the TF-ISF ranking function we are extending it with the estimate of the relevance of the context to the query. It is important to notice that inside the scope of this paper our aim is to show that it is possible to improve the TF-ISF method using local context which was not successful in [1]. In other words we will show improvement of the new method in comparison to the baseline TF-ISF method. As additional evidence we will also compare the new method to the tfmix method presented in [1] that, similarly to our method, tries to improve TF-ISF using surrounding sentences. Tests that show weather our new TF-ISF method with context can outperform other sentence retrieval methods with local context (e.g. language modeling methods with context) are left for future work.

\section{A. Definition of the Ranking Function}

The TF-ISF based ranking function for sentence retrieval is [6], [9]:
$R(s \mid q)=\sum_{t \in q} \log \left(t f_{t, q}+1\right) \log \left(t f_{t, s}+1\right) \log \left(\frac{n+1}{0.5+s f_{t}}\right)$

where

- $t f_{t, q}$ is the number of occurences of term $t$ in question $\mathrm{q}$

- $t f_{t, s}$ is the number of occurrences of term $t$ in sentence $\mathrm{s}$

- $s f_{t}$ number of sentences that contain term $\mathrm{t}$

- $n$ number of sentences in the collection

As the local context of each sentence we are using the previous and next sentence in the same document. We assume that the relevance of a sentence depends partly on the content of the sentence itself and partly on the content of the two neighboring sentences. The relevance of the neighboring sentences again depends partly on their neighbor's content. Using these two assumptions we define the new recursive ranking function for sentence retrieval as follows:

$$
\begin{gathered}
R_{\text {con }}(s \mid q)=(1-\mu) \cdot R(s \mid q)+ \\
\mu \cdot\left[R_{\text {con }}\left(s_{\text {prev }}(s) \mid q\right)+R_{\text {con }}\left(s_{\text {next }}(s) \mid q\right)\right]
\end{gathered}
$$

where $s_{\text {prev }}(s)$ depicts previous sentence of sentence $s$ and $s_{\text {next }}(s)$ next sentence of sentence $s . R_{\text {con }}\left(s_{\text {prev }}(s) \mid q\right)$ and $R_{\text {con }}\left(s_{\text {next }}(s) \mid q\right)$ represent the relevance of the previous and next sentence. $R_{\text {con }}\left(s_{\text {prev }}(s) \mid q\right)$ is by definition 0 if $s$ is first sentence in document and $R_{\text {con }}\left(s_{\text {next }}(s) \mid q\right)$ is by definition 0 if $\mathrm{s}$ is last sentence in document. $\mu$ is a tuning parameter. In our tests in chapter 4 the recursive function calls itself until three previous and three next sentences of the sentence $s$ are involved. In other words three recurrences are used. After that no context is used i.e. $R_{c o n}(s \mid q)=R(s \mid q)$. Of course it is possible to take into account all the neighboring sentences in a document. Tests, showing if it can help to include more than three previous and three next sentences into computation of the relevance of a sentence, are left for future work.

One benefit of our TF-ISF method in comparison to the tfmix method from [1] is the explicit modeling of the relevance of the context (previous and next sentence) to the query $R_{\text {con }}\left(s_{\text {prev }}(s) \mid q\right)+R_{\text {con }}\left(s_{\text {next }}(s) \mid q\right)$ while in [1] parts of the TF-ISF function are replaced with components related to the sentence and to the neighbor sentences $\left(t f_{t, s}\right.$ is replaced with $\alpha \cdot t f_{t, s}+(1-\alpha) t f_{t, \text { context }}$ where $\alpha$ is a tuning parameter). We think that our approach is better because it allows better exploring of the influence of context to the sentence relevance. That is important for our future work where we want to automatically generate a document representation for sentence retrieval. Our function $\left(R_{c o n}(s \mid q)\right)$ can be used to automatically create a useful structured representation of a textual document with a representation of context. Our aim is to use such representations in a web environment where structured document representations are stored at web servers along with the plain text documents. That means that a part of the computation needed for sentence retrieval will be converted to a document representation.

Second benefit of our TF-ISF method are statistically significant improvements according MAP, R-precision and $\mathrm{P} @ 100$ in comparison to the baseline presented in chapter 4 while the tfmix method did not show statistically significant improvements [1]. 


\section{EXPERIMENT}

We tested our new method for sentence retrieval using data from the TREC Novelty tracks. There were three TREC Novelty Tracks in the years from 2003 to 2004 [3], [4], [5]. The task was novelty detection which consists of two subtasks, finding relevant sentences and finding novel sentences. We are only interested in finding relevant sentences which is called sentence retrieval. Sentence retrieval is important part of novelty detection e.g. Allan [6] showed that the performance of novelty detection depends on the quality of the performance of sentence retrieval.

In each of the three Novelty Tracks in the years 2002, 2003 and 2004 the task was as follows: given a topic and an ordered list of documents find relevant and novel sentences. In each Track participants got a set of 50 topics where each topic consisted of titles, descriptions and narratives. They also got a list of mostly relevant documents and a list of sentence level relevance judgments.

In TREC 2002 the topics from ad hoc Tracks were used. To each topic 25 documents were assigned. If the topic had 25 or more relevant documents only 25 relevant documents were used. If the topic had less than 25 documents non-relevant documents were added to reach the number of 25 documents. The assessors marked about $2 \%$ of the sentences relevant.

In TREC 2003 topics where constructed specially for the Novelty track. For every topic 25 relevant documents were chosen. $37.56 \%$ of sentences were judged relevant.

In TREC 2004 between 25 and 100 documents were chosen with 25 of them relevant. $16.2 \%$ of sentences were judged relevant.

An example of a topic from the TREC 2002 Novelty track is shown in Table I.

TABLE I: EXAMPLE OF A TOPIC FROM THE TREC 2002 NOVELTY TRACK

\begin{tabular}{ll}
\hline \hline Title & International Art Crime \\
\hline Description & $\begin{array}{l}\text { Isolate instances of fraud or embezzlement in the } \\
\text { international art trade. }\end{array}$ \\
\hline & $\begin{array}{l}\text { A relevant document is any report that identifies an instance } \\
\text { of fraud or embezzlement in the international buying or } \\
\text { selling of art objects. Objects include paintings, jewelry, } \\
\text { sculptures and any other valuable works of art. Specific } \\
\text { instances must be identified for a document to be relevant; } \\
\text { generalities are not relevant. }\end{array}$ \\
\hline \hline
\end{tabular}

We assume that it is possible to improve the TF-ISF method for sentence retrieval by taking into account the local context of each sentence that consists of the previous (left) sentence and the next (right) sentence (see equation (2)).

To evaluate our new method we compare the baseline TF-ISF method (with ranking function $R(s \mid q)$ ) and the new $\mathrm{TF}_{\text {-ISF }}$ con method we propose (with ranking function $R_{c o n}(s \mid q)$ ). As additional evidence we compare the new TF-ISF ${ }_{\text {con }}$ with the tfmix method from [1].

In the experiments we partially used Rapidminer ${ }^{1}$, an open-source system for data mining with Text Extension ${ }^{2}$ that allows using the vector space model. With Rapidminer all upper cases were transformed to lower case, standard stop words were removed. Stemming was not applied.

\footnotetext{
${ }^{1}$ http://rapid-i.com/content/view/181/196/

${ }^{2}$ http://rapid-i.com/content/view/202/206/
}

We used short queries from the title field. We measured the performance using a series of P@X measures (including the often used P@10) and the standard measures MAP, and R-precision. To compare the difference between two methods we used paired t-test with significance level $\alpha=0.05$.

Our ranking function requires tuning of the parameter $\mu$, so we employed a train-test methodology similar to [1]. We experimented with three training-testing configurations using TREC Novelty track data as follows:

- Training with TREC 2002 and testing with TREC 2003 and TREC 2004

- Training with TREC 2003 and testing with TREC 2002 and TREC 2004

- Training with TREC 2004 and testing with TREC 2002 and TREC 2003

Training was performed to find the value of parameter $\mu$ for which the system shows best performance. During each of the tree trainings (TREC 2002, 2003, 2004) we tried values from $\mu=0.0$ to $\mu=1.0$ in steps of 0.05 . The best value of $\mu$ was fixed in order to apply it to the two remaining data sets. During training we measured the performance of the system by using Mean average precision (MAP). Table II. shows the optimal parameter values for the three datasets.

TABLE II: OPTIMAL $\mu$ VALUES OF TF-ISF CON $_{\text {METHOD }}$

\begin{tabular}{lc} 
& $\mu$ \\
\hline TREC 2002 & 0.2 \\
\hline TREC 2003 & 0.1 \\
\hline TREC 2004 & 0.2 \\
\hline \hline
\end{tabular}

The training of the tfmix method [1] was performed

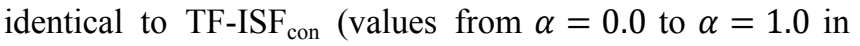
steps of 0.05 were tested). Table III shows optimal parameter values for the three datasets.

\begin{tabular}{cc} 
TABLE III: OPTIMAL $\alpha$ VALUES OF THE TFMIX METHOD \\
\hline TREC 2002
\end{tabular}

The next tables (Table IV, V. and VI) show the results for the three training-testing configurations. Statistically significant differences between the TF-ISF con $_{\text {and the }}$ baseline TF-ISF and between the tfmix and the baseline TF-ISF are marked with an asterisk. Statistically significant differences between the TF-ISF ${ }_{\text {con }}$ and tfmix are marked with a $\dagger$. When it comes to the MAP the tests show significant improvement when using TF-ISF con $_{\text {in }}$ comparison to the baseline TF-ISF and tfmix for each of the tested data sets and values of $\mu$. Also, TF-ISF con shows significant improvement for most of the data sets and values of $\mu$ ( 5 out of 6 ) according R-Precision. When it comes to the TF-ISF con and $\mathrm{P} @ \mathrm{x}$ measures we had altogether 48 measurements with 4 statistically significant improvements (according to P@100) and 44 not statistically significant differences. The tfmix method showed similar performance as in [1] with no statistically significant improvements of the baseline. 
TABLE IV: P@X, MAP AND R-PRECISION FOR TREC 2003 AND 2004, $\mu=0.2, \alpha=0.7$

\begin{tabular}{lcccccc}
\hline \hline & \multicolumn{3}{c}{ TREC 2003 } & & TREC 2004 \\
\hline $\mathrm{P} @ 5$ & TF-ISF & tfmix & TF-ISF $_{\text {con }}$ & TF-ISF & tfmix & TF-ISF $_{\text {con }}$ \\
\hline $\mathrm{P} @ 10$ & 0.7160 & 0.7200 & 0.7200 & 0.4360 & 0.4440 & 0.4560 \\
\hline $\mathrm{P} @ 15$ & 0.6980 & 0.7040 & 0.6980 & 0.4220 & 0.4460 & 0.4460 \\
\hline $\mathrm{P} @ 20$ & 0.7040 & 0.6960 & 0.7000 & 0.4280 & 0.4253 & 0.4320 \\
\hline $\mathrm{P} @ 25$ & 0.6890 & 0.6880 & 0.6980 & 0.4260 & 0.4240 & 0.4230 \\
\hline $\mathrm{P} @ 30$ & 0.6800 & 0.6760 & 0.6864 & 0.4168 & 0.4160 & 0.4176 \\
\hline $\mathrm{P} @ 50$ & 0.6707 & 0.6640 & 0.6747 & 0.4113 & 0.4160 & 0.4147 \\
\hline $\mathrm{P} @ 100$ & 0.6436 & 0.6496 & 0.6556 & 0.4040 & 0.3972 & 0.4028 \\
\hline MAP & 0.6078 & 0.6112 & $0.6184^{*} \dagger$ & 0.3660 & 0.3650 & 0.3716 \\
\hline $\mathrm{R}$-Precision & 0.5764 & 0.5775 & $0.5930^{*} \dagger$ & 0.3252 & 0.3260 & $0.3398^{*} \dagger$ \\
\hline \hline
\end{tabular}

TABLE V: P@X, MAP AND R-PRECISION FOR TREC 2002 AND 2004, $\mu=0.1, \alpha=0.85$

\begin{tabular}{|c|c|c|c|c|c|c|}
\hline & \multicolumn{3}{|c|}{ TREC 2002} & \multicolumn{3}{|c|}{ TREC 2004} \\
\hline & TF-ISF & tfmix & TF-ISF $_{\text {con }}$ & TF-ISF & tfmix & TF-ISF ${ }_{\text {con }}$ \\
\hline $\mathrm{P} @ 5$ & 0.3200 & 0.3360 & 0.3520 & 0.4360 & 0.4400 & 0.4480 \\
\hline $\mathrm{P} @ 10$ & 0.2900 & 0.3140 & 0.3020 & 0.4220 & 0.4420 & 0.4340 \\
\hline P@15 & 0.2973 & 0.2907 & 0.2960 & 0.4280 & 0.4333 & 0.4347 \\
\hline $\mathrm{P} @ 20$ & 0.2760 & 0.2820 & 0.2820 & 0.4260 & 0.4220 & 0.4210 \\
\hline $\mathrm{P} @ 25$ & 0.2648 & 0.2776 & 0.2752 & 0.4168 & 0.4192 & 0.4232 \\
\hline $\mathrm{P} @ 30$ & 0.2587 & 0.2680 & 0.2680 & 0.4113 & 0.4160 & 0.4187 \\
\hline P@50 & 0.2416 & 0.2416 & 0.2488 & 0.4040 & 0.3996 & 0.3988 \\
\hline $\mathrm{P} @ 100$ & 0.1904 & 0.1934 & $0.2146^{* \dagger}$ & 0.3660 & 0.3672 & 0.3714 \\
\hline MAP & 0.1952 & 0.1973 & $0.2315^{* \dagger}$ & 0.3252 & 0.3258 & $0.3392 * \dagger$ \\
\hline R-Precision & 0.2414 & 0.2448 & $0.2666^{* \dagger}$ & 0.3376 & 0.3403 & $0.3473 * \dagger$ \\
\hline
\end{tabular}

TABLE VI: P@X, MAP AND R-PRECISION FOR TREC 2002 AND 2003, $\mu=0.2, \alpha=0.6$

\begin{tabular}{lcccccc}
\hline \hline & \multicolumn{3}{c}{ TREC 2002 } & & TREC 2003 \\
\hline & TF-ISF & tfmix & TF-ISF $_{\text {con }}$ & TF-ISF & tfmix & TF-ISF $_{\text {con }}$ \\
\hline $\mathrm{P} @ 5$ & 0.3200 & 0.3200 & 0.3480 & 0.7160 & 0.7240 & 0.7200 \\
\hline $\mathrm{P} @ 10$ & 0.2900 & 0.3140 & 0.3040 & 0.6980 & 0.6860 & 0.6980 \\
\hline $\mathrm{P} @ 15$ & 0.2973 & 0.3000 & 0.3000 & 0.7040 & 0.6947 & 0.7000 \\
\hline $\mathrm{P} @ 20$ & 0.2760 & 0.2910 & 0.2880 & 0.6890 & 0.6830 & 0.6980 \\
\hline $\mathrm{P} @ 25$ & 0.2648 & 0.2784 & 0.2792 & 0.6800 & 0.6800 & 0.6864 \\
\hline $\mathrm{P} @ 30$ & 0.2587 & 0.2680 & 0.2727 & 0.6707 & 0.6667 & 0.6747 \\
\hline $\mathrm{P} @ 50$ & 0.2416 & 0.2440 & 0.2496 & 0.6436 & 0.6508 & 0.6556 \\
\hline $\mathrm{P} @ 100$ & 0.1904 & 0.1934 & $0.2154 * \dagger$ & 0.6078 & 0.6100 & $0.6184 * \dagger$ \\
\hline MAP & 0.1952 & 0.1971 & $0.2322^{*} \dagger$ & 0.5764 & 0.5773 & $0.5930^{*} \dagger$ \\
\hline R-Precison & 0.2414 & 0.2451 & $0.2672 * \dagger$ & 0.5457 & 0.5483 & $0.5725^{*} \dagger$ \\
\hline \hline
\end{tabular}

To achieve a better insight into the performance of the new method regarding R-precision and P@x measures we put the data of all three TREC-s together. This time we do not have a training data set to pick the best value for $\mu$. For that reason we report the results for a whole range of values. The results are shown in Table VII. Again significant differences to the baseline are marked with an asterisk.

This time we can see improvements for a whole range of $\mu$-values $(\mu=0.1-0.3)$ when it comes to the MAP and R-Precision. At the same time we do not have significant differences according the $\mathrm{P} @ \mathrm{x}$ values (except for $\mathrm{P} @ 100)$. When choosing higher values of $\mu(\mu=0.4, \mu=0.5)$ we start to get significantly worse results according to some of the $\mathrm{P} @ \mathrm{x}$ measures and stop getting significantly better results according to MAP and R-Precision. This scenario was expected because there must be a threshold value of $\mu$ at which the influence of the neighboring sentences is too high.

We can see from the Table VII that it is quite easy to find a value of $\mu$ that improves the baseline according to MAP and $\mathrm{R}$-Precision and at the same time gives competitive results according to reported $\mathrm{P} @ \mathrm{x}$ measures. It should be noted that we also have significant better results according $\mathrm{P} @ 100$ measures for $\mu=0.1, \mu=0.2$ and $\mu=0.3$. 
TABLE VII: P@X, MAP AND R-PRECISION FOR THE COMBINED DATA SETS OF TREC 2002, TREC 2003 AND TREC 2004

\begin{tabular}{lcccccc}
\hline \hline & TF-ISF & \multicolumn{5}{c}{ TF-ISF $_{\text {con }}$} \\
\cline { 3 - 7 } & & $\mu=0.1$ & $\mu=0.2$ & $\mu=0.3$ & $\mu=0.4$ & $\mu=0.5$ \\
\hline $\mathrm{P} @ 5$ & 0.4907 & 0,5107 & 0,5080 & 0,4987 & 0,4853 & 0,4600 \\
\hline $\mathrm{P} @ 10$ & 0.4700 & 0,4847 & 0,4827 & 0,4787 & 0,4633 & 0,4367 \\
\hline $\mathrm{P} @ 15$ & 0.4764 & 0,4782 & 0,4773 & 0,4702 & 0,4547 & $0,4409^{*}$ \\
\hline $\mathrm{P} @ 20$ & 0.4637 & 0,4663 & 0,4697 & 0,4657 & 0,4537 & $0,4353^{*}$ \\
\hline $\mathrm{P} @ 25$ & 0.4539 & 0,4629 & 0,4611 & 0,4573 & 0,4480 & $0,4291^{*}$ \\
\hline $\mathrm{P} @ 30$ & 0.4469 & 0,4549 & 0,4540 & 0,4476 & 0,4431 & $0,4187^{*}$ \\
\hline $\mathrm{P} @ 50$ & 0.4297 & 0,4337 & 0,4360 & 0,4353 & 0,4189 & $0,3999^{*}$ \\
\hline $\mathrm{P} @ 100$ & 0.3881 & $0,4014^{*}$ & $0,4018^{*}$ & $0,3974^{*}$ & 0,3903 & $0,3762^{*}$ \\
\hline MAP & 0.3656 & $0,3885^{*}$ & $0,3883^{*}$ & $0,3850^{*}$ & $0,3771^{*}$ & 0,3634 \\
\hline R-Prec. & 0.3749 & $0,3944^{*}$ & $0,3951^{*}$ & $0,3914^{*}$ & 0,3815 & 0,3683 \\
\hline \hline
\end{tabular}

The measures MAP and R-precision for which our new method shows better performance are recall oriented. This is identical to the improvements of including context into language modeling framework [1]. Including context promotes sentences that don't have many terms in common with the query but have some terms in common in the context which increases recall. Recall is important for the application scenario presented in [3], [1]. In the scenario a user uses a smart "next" button which allows him to walk down a ranked list of documents by highlighting only relevant (and novel) sentences. Having access to all relevant documents is also important for multi-document summarization [1]. At the same time when it comes to the reported precision oriented measures (P@5-P@100)we have competitive performance (except for $\mathrm{P} @ 100$ where we have significant improvements) so we do not have to worry when performing tasks that require high precision like question answering.

Another interesting point is that our method also shows improved performance according to MAP and R-precision, and $\mathrm{P} @ 100$ when using even smaller values of $\mu$ than 0.1 . For example we tried $\mu=0.01$ and got significantly better results. The reason may lie in the fact that the baseline TF-ISF ranking function produces the same scores for multiple sentences. At such situations additional evidence, no matter how small it is, from the neighbor sentences can help to better rank the sentences.

\section{CONCLUSION}

At the beginning of this paper we presented the state of the art and most successful methods for sentence retrieval, the TF-ISF method and the language modeling approach with local context. It was stated that it is important to take into account the local context of sentences because of the word mismatch problem and additional content that is related to the sentence. An earlier work tried to improve the TF-ISF method using some context but without statistically significant improvement. Therefore, the novel TF-ISF method with a recursive ranking function was proposed that in a new way and successfully extends the TF-ISF method to take into account the local context of sentence. Precisely the local context is considered to be the previous and the next sentence. The method was compared against the TF-ISF baseline and the tfmix method (an earlier try to include context into TF-ISF presented in [1]) and showed statistically significant improvements according the MAP and R-Precision measures and competitive results according a series of $\mathrm{P} @ \mathrm{x}$ measures. One additional benefit of the new $\mathrm{TF} \mathrm{ISF}_{\text {con }}$ method is that it models explicitly the relevance of the context to the query which allows better exploration of the influence of the context to sentence relevance. In future works we plan to automatically generate a useful structured representation of a textual document with a representation of context capable of sentence retrieval and that can be stored on web servers.

\section{REFERENCES}

[1] R. T. Fernandez, D. E. Losada, L. A. Azzopardi, "Extending the language modeling framework for sentence retrieval to include local context," Information Retrieval, vol. 14, no. 4, pp. 355-389, 2010.

[2] V. G. Murdock, "Aspects of sentence retrieval," $\mathrm{PhD}$ thesis, University of Massachusetts Amherst, 2006.

[3] D. Harman, Overview of the TREC 2002 novelty track, in Proceeding of the Eleventh Text Retrieval Conference (TREC), 2002.

[4] I. Soboroff and D. Harman, Overview of the TREC 2003 novelty track, in Proceeding of the Eleventh Text Retrieval Conference (TREC), 2003.

[5] I. Soboroff, Overview of the TREC 2004 novelty track, in Proceeding of the Eleventh Text Retrieval Conference (TREC), 2004.

[6] J. Allan, C. Wade, A. Bolivar, Retrieval and novelty detection at the sentence level, in Proceedings of the 26th ACM international conference on research and development in information retrieval (SIGIR 2003) , pp. 314-321, 2003.

[7] H. P. Zhang, H. B. Xu, S. Bai, B. Wang, and X. Q. Cheng, "Experiments in TREC 2004 novelty track at CAS-ICT," in Proc. TREC-2004, 2004

[8] D. E. Losada and R. T. Fernandez, Highly frequent terms and sentence retrieval, in Proceedings of the 14th String processing and information retrieval symposium (SPIRE 2007), Lecture Notes in Computer Science, pp. 217-228, Santiago de Chile, Chile: Springer, 2007.

[9] D. E. Losada, A study of statistical query expansion strategies for sentence retrieval, in Proceedings SIGIR 2008 workshop on focused retrieval (question answering, passage retrieval, element retrieval), Singapore: ACM, 2008.

[10] R. T. Fernandez, D. E. Losada, "Using opinion-based features to boost sentence retrieval," in Proceedings of the ACM 18th conference on information and knowledge management (CIKM 2009), pp. 1617-1620, Hong Kong, China: ACM, 2009.

[11] J. Ponte and W. B. Croft, "A language modeling approach to information retrieval," in Proceedings of the 21st Annual Conference on Research and Development in Information Retrieval (ACM SIGIR), 1998.

[12] M.-F. Tsai and H.-H Chen, "Some Similarity Computation Methods in Novelty Detection," in Proceedings of the Eleventh Text Retrieval Conference. Gaithersburg, NIST Special Publication, pp. 500-251, 2002. 


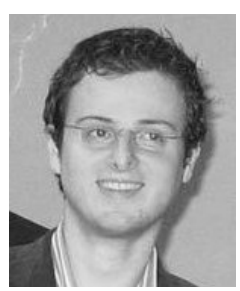

Alen Doko was born on 1982 in Mostar, Bosnia and Herzegovina. He received his Graduate Engineer Degree in 2007. Since 2007 he has been a Ph.D student.

$\mathrm{He}$ is also a Business and Analytical Information Systems Associate at JP Croatian Telecommunication d.o.o. Mostar, Mostar, Bosnia and Herzegovina. His research interests include Semantic Web, Information Retrieval and Sentence Retrieval. He is author and coauthor of two scientific papers.

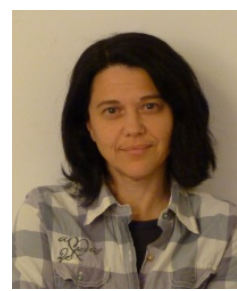

Maja Štula is associated professor of computer science at the Faculty of Electrical Engineering, Mechanical Engineering and Naval Architecture, University of Split. She received her B.S.E.E. in 1996. , M.S.E.E. in 2001. and Ph.D. title in 2005, all from FESB, University of Split.

Her current position is associated professor of computer science at FESB, University of Split. Previous positions include companies THT (Croatian Telecom) and Infin d.o.o. where she worked as system designer and developer. She is employed by FESB from 1998. She is author or coauthor of more than 40 scientific papers, mainly in the multi-agent and fuzzy cognitive map fields.
Dr. Štula's research interests include design, analysis and implementation of intelligent technologies from areas of multi-agent systems, information retrieval, qualitative modeling with fuzzy cognitive maps and intelligent environment protection. She is IEEE member.

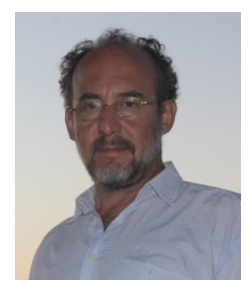

Darko Stipaničev is professor of computer science and automatic control at Faculty of Electrical Engineering, Mechanical Engineering and Naval Architecture (FESB), University of Split. He received his Ph.D. title in Electrical Engineering from University of Zagreb in 1989.

His current position is senior full professor at FESB, University of Split from 2002. Previous positions include researcher position at company Koncar, Zagreb 1977-1980. From 1981. he is employed by FESB, University of Split.

He is also Head of center for wildfire research and Chair of department for Modeling and Intelligent Computer Systems. He is author or coauthor of more than 100 scientific papers.

Dr. Stipaničev's research interest include complex systems modeling and control, intelligent systems analyses and design, digital image analyses, advanced Internet technologies and recently, the application of ICT in environmental protection, particularly wildfire prevention and management. He is IEEE member. More details on http://laris.fesb.hr/dstip-e.html. 